\title{
Herpes zoster-associated aseptic arthritis in adult patients: A case report
}

\section{Dear Editor,}

Aseptic arthritis can often be associated with virusesin the Singapore context-dengue or chikungunya viruses. The association of varicella-zoster virus (VZV) with arthritis however, is rare. Few cases of aseptic arthritis associated with VZV have been documented in the paediatric literature in the past 50 years and fewer still in adults. To date, the exact mechanism, clinical presentation and treatment have not been well described in the literature. We describe here a case of aseptic arthritis associated with VZV reactivation and present a review of the published literature.

In December 2017, a 79-year-old Chinese man presented to our rheumatology clinic with left hand pain and swelling. Prior to this, the man had no history of joint complaints. He developed herpes zoster over the left C6 dermatome in September 2017. No antiviral treatment was prescribed when the patient first developed herpes zoster. Pain was worse when carrying or gripping objects but not worse in the morning. Stiffness lasted the whole day. The symptoms had persisted without improvement over 4 months and he remained unable to perform most of his daily activities with his left hand. He had no constitutional symptoms of fever, fatigue, anorexia or weight loss, and systemic review was otherwise unremarkable. His past medical history was significant for hypertension, atrial fibrillation, severe tricuspid regurgitation and moderate ischaemic mitral regurgitation.

Physical examination revealed a well-nourished elderly man, with tenderness over the proximal interphalangeal joints of his left index and middle finger. The entire left hand was puffy and oedematous, with a greatly reduced range of movement. He was unable to straighten his fingers fully or make a full fist, but clinical synovitis was not readily appreciated due to the swelling. His wrist was not swollen, and there were no signs of carpal tunnel syndrome. There was no remnant cutaneous sign of his previous herpes zoster, and no other joint swellings. Laboratory investigations showed negative rheumatoid factor and anti-cyclic citrullinated protein antibodies (Table 1). Anti-VZV immunoglobulin $\mathrm{M}$ and immunoglobulin $\mathrm{G}$ were not done as the herpes zoster had resolved 4 months ago. X-ray of the hand was normal with no evidence of chrondrocalcinosis. Ultrasound revealed grade 2-3 synovial hypertrophy of the proximal interphalangeal joints of the right thumb, left index, middle and little fingers, as well as mild osteoarthritic changes in both hands. There was no power Doppler signal visualised on ultrasound. Joint aspiration could not be done because of an absence of joint effusion on clinical examination and ultrasound.

He was diagnosed with an asymmetrical oligoarthritis of the hand, possibly zoster-associated arthritis. Due to the long duration of his symptoms, he was treated empirically with hydroxychloroquine $400 \mathrm{mg}$ daily and a short course of prednisolone (10mg for 1 week, followed by $5 \mathrm{mg}$ for 1 week). His symptoms and signs ameliorated 4 months later. The stiffness and swelling improved, his joints were no longer tender, and he was once again able to make a full fist with his left hand. Hydroxychloroquine was discontinued and the patient remained well.

From 1968-1993, 7 articles describing 8 cases of zoster-associated arthritis were reported in adults. Subsequently, only 1 case was reported in 2017. In contrast, although rare, arthritis is a well-recognised complication of primary VZV infection in otherwise healthy, immunocompetent children. From our literature review, the following clinical features are present in herpes zoster-associated arthritis: (1) small and large joint involvement; (2) synchronous onset; (3) joint involvement corresponding to dermatomal involvement; (4) neutrophil predominance on synovial fluid analysis; and (5) evidence of VZV in synovial fluid on laboratory testing (Table 1). As with most classification criteria in rheumatology, the presence of some but not all of the above features is suggestive of zosterassociated arthritis.

The demographic, clinical and biologic characteristics of the 9 patients are presented in Table 1. The median age was 67 years and 4 of them were male. Three patients were considered immunocompromised2 had long-standing rheumatoid arthritis of more than 10 years and 1 had non-Hodgkin's lymphoma. Five had monoarthritis of the shoulder, hips or knees, with the herpes zoster eruption over the corresponding joint distribution. The median age of 67 years corresponds with the common age of VZV reactivation. ${ }^{1-3}$

Joint involvement usually starts at the time of diagnosis of herpes zoster, the median onset time being 0 days (interquartile [IQR] range, -6.0-1.0) 


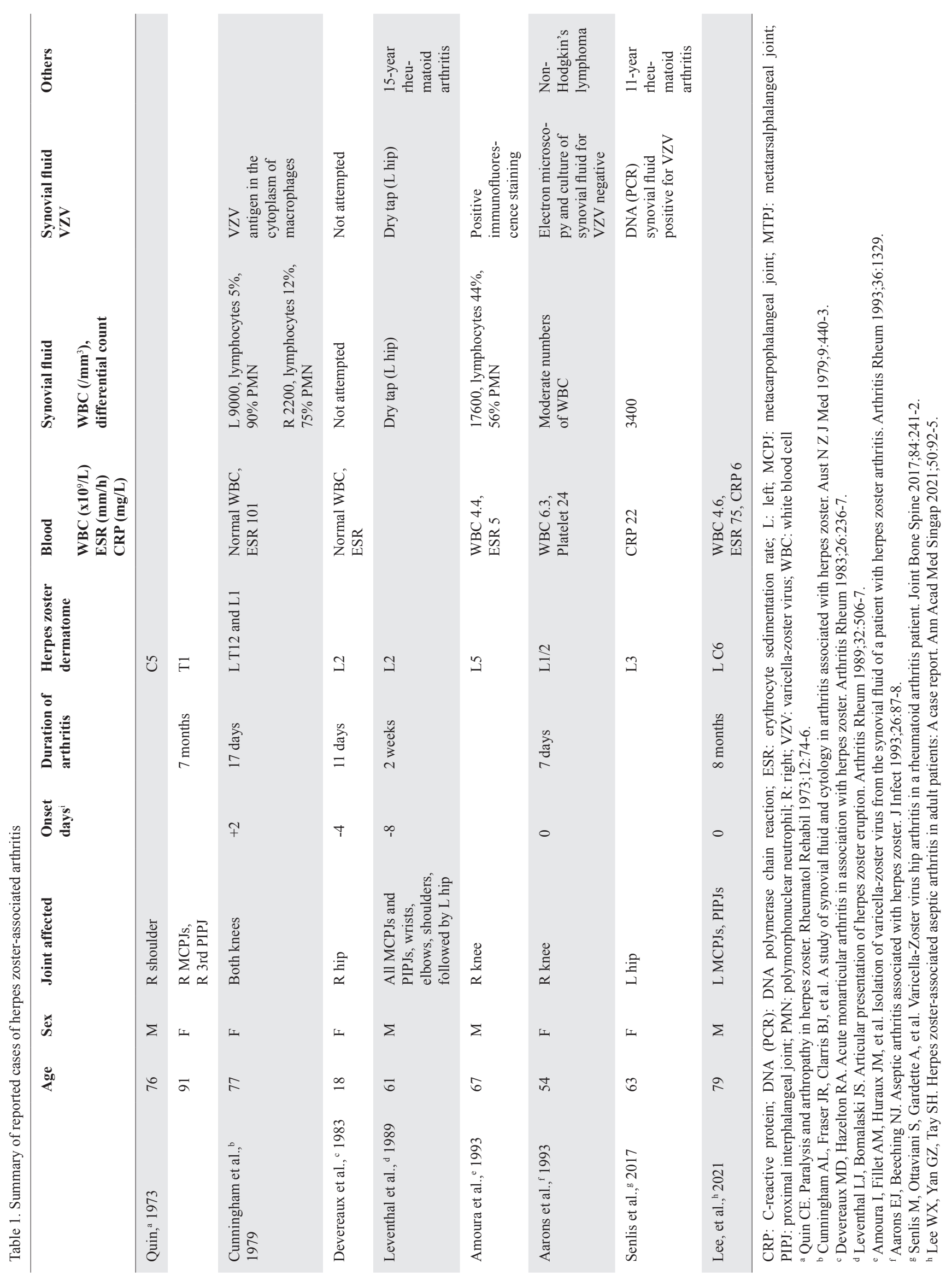


(Table 1). In terms of symptom duration, it was interesting to note that although most cases of zosterassociated arthritis resolved in a median of 2.2 weeks (IQR 1.4-35.0), 1 patient experienced persistent arthritis that lasted for 7 months. ${ }^{4}$ Our patient was similar in that he experienced joint swelling as well as skin and subcutaneous oedema of his left hand that took 8 months to resolve. It is worth noting that despite prolonged arthritis, all cases that have been reported thus far resolved eventually. Other viruses like parvovirus B19 can also induce chronic inflammatory arthritis that lasts for months, but mainly affecting women rather than men. ${ }^{5}$

In the immunopathogenesis of herpes zosterassociated arthritis, it has been noted that there is a predominance of neutrophils in the synovial fluid rather than lymphocytes. This is in contrast to primary VZV-associated arthritis and other virus-associated arthritis, which is associated with a predominance of lymphocytes. This may have implications when speculating on the role of neuro-immunological communication in the occurrence of herpes zosterassociated arthritis. It has been described that nociceptors suppress the recruitment and surveillance of neutrophils. ${ }^{6}$ Pain in herpes zoster and post-herpetic neuralgia is due to inflammation-induced sensitisation of nociceptor endings and later, deafferentation due to destruction of the sensory neutrons in the affected dorsal root ganglion. ${ }^{7}$ Hence, the involvement of the dorsal root ganglion and subsequent destruction of nociceptors in herpes zoster may explain the neutrophilic predominance in herpes zoster-associated arthritis and not arthritis associated with primary VZV infection.

The joints are innervated by peripheral sensory afferents originating from spinal sensory neurons, whose cell bodies originate within the dorsal root ganglia. ${ }^{6}$ This suggests that VZV may spread along the nerves to the joints, similar to how it spreads to the skin in disseminated herpes zoster. In some cases where pain rather than swelling was the predominant presentation, joint pain may also be contributed largely by nerve root involvement. However, some of the patients had widespread joint involvement beyond the dermatome affected by herpes zoster reactivation. This suggests a different pathophysiology, perhaps a generalised inflammatory response to viral immune activation.

Similar to the syndrome of aseptic meningitis, we elected to adopt the terminology of "aseptic" to describe zoster-associated arthritis since there is synovial fluid pleocytosis in the absence of a positive Gram stain and culture. ${ }^{8}$ Of the 9 cases, 5 had synovial fluid assessment, and 3 were positive for $\mathrm{VZV}$, although all 3 were tested using different methods. Polymerase chain reaction (PCR) test is the most sensitive method for the detection of VZV DNA in clinical samples (e.g. fluid from vesicles, cerebrospinal fluid) and together with its rapid turnaround time, has largely superseded traditional methods of obtaining a microbiological diagnosis such as viral culture and immunofluorescent techniques. ${ }^{9}$

Antiviral therapy is recommended for use in herpes zoster, ideally within the first 72 hours of infection. Immune complications of herpes zoster requiring immunosuppression have also been reported. The use of adjuvant glucocorticoids in complicated herpes zoster infections (e.g. herpes zoster ophthalmicus and Ramsay Hunt Syndrome) as well as immunologic complications of VZV (e.g. transverse myelitis) has been reported to be beneficial. ${ }^{10}$ Our patient received a short course of oral prednisolone and hydroxychloroquine with good response. Hence, immunosuppression can be considered for use in treating zoster-associated arthritis once septic arthritis has been appropriately excluded. It is important to consider other differential diagnoses such as septic arthritis, crystal arthritis and flare of existing inflammatory arthritis, as zoster-associated arthritis is relatively rare. Arthrocentesis can also be helpful in distinguishing between these differential diagnoses. ${ }^{11}$

In summary, in patients with herpes zoster-associated arthritis, we suggest PCR test on the synovial fluid to look for VZV DNA, treatment with antiviral therapy such as acyclovir, and to consider the use of glucocorticoids if not otherwise contraindicated. Most cases are self-limiting but chronic inflammatory arthritis requiring prolonged immunosuppression may be required.

\section{REFERENCES}

1. Cocchio S, Baldovin T, Furlan P, et al. Cross-sectional study on hospitalizations related to herpes zoster in an Italian region, 2008-2016. Aging Clin Exp Res 2019;31:145-50.

2. Pham MA, Bednarczyk RA, Becker ER, et al. Trends in U.S. Community hospitalizations due to herpes zoster: 2001-2015. Vaccine 2019;37:882-8.

3. Marra F, Chong M, Najafzadeh M. Increasing incidence associated with herpes zoster infection in British Columbia, Canada. BMC Infect Dis 2016;16:589.

4. Quin CE. Paralysis and arthropathy in herpes zoster. Rheumatol Rehabil 1973;12:74-6.

5. Woolf AD, Campion GV, Chishick A, et al. Clinical manifestations of human parvovirus B19 in adults. Arch Intern Med 1989; 149:1153-6. 
6. Baral P, Umans BD, Li L, et al. Nociceptor sensory neurons suppress neutrophil and $\gamma \delta \mathrm{T}$ cell responses in bacterial lung infections and lethal pneumonia [published correction in Nat Med 2018;24:1625-26]. Nat Med 2018;24:417-26.

7. Devor M. Rethinking the causes of pain in herpes zoster and postherpetic neuralgia: the ectopic pacemaker hypothesis. Pain Rep 2018;3:e702.

8. Shukla B, Aguilera EA, Salazar L et al. Aseptic meningitis in adults and children: diagnostic and management challenges. J Clin Virol 2017;94:110-4.

9. Arvin AM. Varicella-zoster virus. Clin Microbiol Rev 1996; 9:361-81.

10. Coulson S, Croxson GR, Adams R, et al. Prognostic factors in herpes zoster oticus (ramsay hunt syndrome). Otol Neurotol 2011;32:1025-30

11. Chong YY, Fong KY, Thumboo J. The value of joint aspirations in the diagnosis and management of arthritis in a hospital-based rheumatology service. Ann Acad Med Singap 2007;36:106-9.
Weixian Lee, ${ }^{1} M B B S$, Gabriel Zherong Yan, ${ }^{2} M B B S$, Sen Hee Tay, ${ }^{3,4}{ }_{M B B S}$

\footnotetext{
1 Department of Medicine, Division of Rheumatology, Ng Teng Fong General Hospital, Singapore

${ }^{2}$ Department of Medicine, Division of Infectious Diseases, National University Hospital, Singapore

${ }^{3}$ Department of Medicine, Yong Loo Lin School of Medicine, National University of Singapore, Singapore

${ }^{4}$ Department of Medicine, Division of Rheumatology, National University Hospital, Singapore
}

Correspondence: Dr Weixian Lee, Ng Teng Fong General Hospital, Tower A, Level 8 Office, 1 Jurong East Street 21, Singapore 609606. Email: weixian_lee@nuhs.edu.sg 